\title{
SOLDATENKAISER, SCRIPTORES HISTORIAE AUGUSTAE UND SCHOLLENBINDUNG. BEMERKUNGEN ZU KLAUS-PETER JOHNES KAISER, KONSULN UND KOLONEN (2007) UND SEINEM HISTORISCHEN GESAMTWERK*
}

Klaus-Peter Johne, Kaiser, Konsuln und Kolonen. Studien zu Kaiserzeit und Spätantike [= Studien zur Geschichtsforschung des Altertums 15]. Hamburg: Verlag Dr. Kovač, 2007, 272 S., ISBN 978-3-8300-2707-2.

\section{RAPHAEL BRENDEL}

\begin{abstract}
Soldier emperors, Scriptores historiae Augustae and serfs tied to the land:

Remarks on Klaus-Peter Johne's Kaiser, Konsuln und Kolonen (2007) and his historical work

This is a detailed discussion of the volume of collected studies of the wellknown classicist Klaus-Peter Johne which was published in 2007. It offers a summary of the individual papers, a discussion of their contents, and several additions to the list of publications, which is a useful bibliographical tool. Together with a short assessment of Johne's contributions in the context of the scholarship of the German Democratic Republic, some general methodical remarks are offered on the concept of a volume of collected studies.
\end{abstract}

Keywords: colonate; Elbe; Germans; Historia Augusta; Klaus-Peter Johne; Prosopographia Imperii Romani; prosopography; Tacitus (emperor)

Der Band, dem die hier gebotenen Zeilen gewidmet sind, hat bisher, soweit ich sehe, keine Beachtung in den Rezensionsteilen altertumswissenschaftlicher Fachzeitschriften gefunden. Das alleine wäre noch keine Rechtfertigung für eine derart späte Rezension, wohl aber die Person des Autors. Klaus-Peter Johne, zuletzt an der Humboldt-Universität in Berlin tätig, gilt mit Recht als einer der besten Kenner der Zeit der Soldatenkaiser

\footnotetext{
Seitenangaben ohne weitere Angabe beziehen sich immer auf diesen Band. Mein Dank gilt den Herausgebern der Zeitschrift, die sich auf das Wagnis dieses etwas unkonventionellen Beitrages eingelassen haben, dem Verlag Dr. Kovač, der trotz des seit der Publikation des Bandes vergangenen langen Zeitraumes dennoch bereitwillig ein Rezensionsexemplar zur Verfügung stellte, und Thomas Banchich (Canisius College), der die ins Englische übersetzten Teile durchgesehen hat.
} 
(235-284 n. Chr.) und ist der Herausgeber des wichtigsten und entsprechend vielbeachteten Handbuches zu dieser Epoche (siehe auch die Liste der Rezensionen dazu unten). Daneben hat er sich wiederholt zu dem Problem der Historia Augusta geäußert, jener rätselhaften Sammlung von Biographien der Kaiser von Hadrian bis Carinus (117-285 n. Chr.), deren Abfassungszeit und Zweck bis heute nicht zufriedenstellend geklärt ist und der sich Johne bereits in seiner Dissertation widmete. Zwei weitere seiner Forschungsschwerpunkte sind das Phänomen des Kolonats in der römischen Geschichte und die Beziehung zwischen Rom und den germanischen Völkern. Zuletzt war Johne auch Mitarbeiter der Prosopographia Imperii Romani (PIR), jenes unersetzlichen prosopographischen Standardwerkes für die ersten drei Jahrhunderte der Kaiserzeit. ${ }^{1}$ Ein Band, in dem eine Reihe von Aufsätzen des verdienten Forschers zu diesen zentralen Themen erneut abgedruckt sind, darf also von vornherein eine gewisse Bedeutung beanspruchen. Kann er den sich daraus ergebenden Erwartungen auch gerecht werden?

Zunächst zum Inhalt: Den Aufsätzen Johnes vorangestellt sind ein Vorwort (S. 7-8) und eine Kurzbiographie Johnes (S. 9-14), die beide von dem Herausgeber Udo Hartmann, selbst ein exzellenter Kenner der Soldatenkaiserzeit, verfasst wurden. Den insgesamt 22 Aufsätzen (S. 17-262), unterteilt nach den fünf Forschungsschwerpunkten Johnes (S. 17-59 Prosopographie; S. 61-108 Germanien; S. 109-147 Soldatenkaiser; S. 149-196 Kolonat; S. 197-262 Historia Augusta) folgen ein Gesamtverzeichnis (fast) aller Schriften Johnes (S. 263-270), auf das noch ausführlicher zurückzukommen sein wird, und eine Liste der Erstpublikationen der in den Band aufgenommenen Aufsätze (S. 271-272).

Dem Vorwort (S. 7-8) sind der Anlass und die Konzeption des Bandes zu entnehmen: Publiziert wurde er anlässlich des 65. Geburtstages von Johne und umfasst „Beiträge, die für die weiteren Forschungen auf den entsprechenden Themenfeldern wesentlich waren und von bleibender Bedeutung sind“ (S. 7). Die insgesamt 22 Aufsätze stammen aus den Jahren 1967 bis 2006, sind thematisch angeordnet (eine Systematik der Anordnung der einzelnen Aufsätze innerhalb der Oberthemen wird allerdings nicht benannt und ist auch nicht ersichtlich) und fast vollkommen unverändert, wenngleich in neuer Setzung abgedruckt. Geändert gegenüber den Erstpublikationen wurden nur kleinere Formalien: Endnoten sind in Fußnoten umgewandelt, die Anmerkungen in jedem Aufsatz durchgezählt, lateinische Begriffe und Zitate kursiv gesetzt, Kapitälchen in normale Buchstaben umgewandelt und die ursprüngliche Paginierung der Erstpublikationen ist ergänzend beigefügt.

Die Kurzbiographie („Klaus-Peter Johne - Stationen eines Forscherlebens“, S. 9-14) informiert zuverlässig über die Entwicklung der Karriere und Forschung Johnes und dürfte vor allem Erforschern der Wissenschaftsgeschichte einen guten ersten Anlaufpunkt bieten. Lediglich eine Beobachtung: S. 9 wird Wolfgang Seyfarth, der wichtigste akademische Lehrer Johnes und Erstgutachter seiner Dissertation, als „Althistoriker“ bezeichnet. Selbst wenn das formal korrekt sein sollte, dürfte es nicht ganz den Tatsachen entsprechen. Zum einen beendete Johne sein Studium bei Seyfarth zunächst mit dem Abschluss eines Fachlehrers für Latein und Griechisch sowie dem eines Diplom-Philologen (S. 9); Seyfarths eigenes Studium weiterhin umfasste sowohl Klassische Philologie

1 Ausführlich zur Geschichte dieses Unternehmens handelt nun der Anm. 11 zitierte Beitrag von Werner Eck. 
als auch Geschichte; ${ }^{2}$ zuletzt stammt von Seyfarth eine Vielzahl philologischer Beiträge, von denen der wichtigste seine Edition des Ammianus Marcellinus ist.

Der erste thematische Block ("Studien zur Prosopographie der römischen Kaiserzeit“, S. 17-59) ist den Beiträgen Johnes zur Prosopographie gewidmet. Dabei wurde allerdings keiner seiner zahlreichen Artikel in der PIR (siehe dazu unten) oder der dieselbe ergänzende Aufsatz zu dem Kaiserbiographen Marius Maximus (S. 264) berücksichtigt, sondern bei den insgesamt fünf aufgenommenen Studien handelt es sich meist um breiter gefasste Überblicke zur Geschichte und Methodik der Prosopographie.

Bei „100 Jahre Prosopographia Imperii Romani“ (S. 17-24) handelt es sich ursprünglich um einen Vortrag anlässlich der Emeritierung von Leiva Petersen, der langjährigen Herausgeberin der PIR (zu ihr S. 10), gehalten am 29. November 1972. Johne bietet hierin einen Überblick über die Systematik und Geschichte der PIR. Der Aufsatz ist für den eigentlichen Althistoriker ebenso von Interesse wie für den Wissenschaftshistoriker, da einerseits Aufbau und Funktion der PIR diskutiert und das Werk mit verwandten Projekten sowie die beiden Auflagen der PIR miteinander verglichen werden, andererseits auch die Geschichte der PIR von ihren Anfängen (Anregung durch Mommsen und die erste Bearbeitung durch Dessau, Klebs und von Rohden) bis in die (damalige) Gegenwart nachgezeichnet wird.

Der nächste Beitrag („Die Scriptores historiae Augustae und die Prosopographia Imperii Romani. Zum Beginn der modernen Historia-Augusta-Forschung vor 100 Jahren“, S. 25-34) - ein Vortrag, der den Besuch Jan Burians in Berlin auf einer Veranstaltung zum hundertjährigen Jubiläum der Entdeckungen Dessaus zum Anlass hatte und dann auf dem Genfer Kolloquium zur Historia Augusta (9.-12. Mai 1991) gehalten und im entsprechenden Kongressband publiziert wurde - weist starke Überschneidungen mit dem vorhergehenden auf, konzentriert sich jedoch vor allem auf die Bedeutung der prosopographischen Forschungen Hermann Dessaus für seine Erkenntnis, dass die Historia Augusta nicht in der Zeit geschrieben sein kann, in der sie geschrieben zu sein vorgibt.

Der Aufsatz zu „Vergleich und Analogie in der prosopographischen Methode“ (S. 35-43), zuerst erschienen in einem Themenband einer Zeitschrift zu „Vergleich und Analogieschluss in den Altertumswissenschaften", 3 befasst sich in allgemeinerer Form mit der Wissenschaft der Prosopographie. Johne erläutert hierin deren Möglichkeiten und Grenzen (so kann auf diesem Wege etwa weder die Macht des Senates noch die Opposition gegen den Kaiser im Detail erfasst werden) und demonstriert die Fortschritte der prosopographischen Forschung exemplarisch an einem Beispiel aus dem damals aktuellsten Faszikel der PIR.

„Die Publii Martii - eine prosopographische Studie“ (S. 45-52), ursprünglich ein Vortrag auf dem VII. Internationalen Kongress für griechische und lateinische Epigraphik (Constanţa, 9.-15. September 1977) behandelt die senatorischen Familien der Martii und bietet eine nützliche wie eingehende Belegsammlung und Statistik zu selbigen.

2 Siehe dazu den Lebenslauf seiner Dissertation Untersuchungen zur Kompositionsweise des Tacitus in den Historien, Diss., Berlin, 1934, unpaginierte S. 64.

3 Der Kongressbericht (der allerdings nicht auf Johnes Beitrag eingeht): Bernhard Rink, „Vergleich und Analogieschluß in den Altertumswissenschaften (3. bis 5. November 1987 in Heiligendamm)", Jahrbuch für Wirtschaftsgeschichte 26/3, 1988, S. 249-251. 
Ebenfalls in dem Band berücksichtigt ist der Beitrag „Zu den Siegernamen der Kaiser Marc Aurel und Commodus“ (S. 53-59), bei dem es sich um die erste Publikation Johnes (außer seiner ungedruckten Diplomarbeit) überhaupt handelt; erschienen ist der Aufsatz in einem Heft der Zeitschrift Klio, das Werner Hartke, einem der akademischen Lehrer Johnes und Zweitgutachter von dessen Dissertation, anlässlich seines 60. Geburtstages am 1. März 1967 gewidmet ist. Behandelt werden im Wesentlichen zwei Themen: Zum einen ordnet Johne zwei in ihrer Datierung unsichere Papyrusfragmente der 1963 publizierten Papyri Iandanae in die Zeit des Commodus ein; daneben kann er durch die allgemeine Untersuchung der Titulatur des Kaisers Marcus Aurelius zeigen, dass dessen Anweisung, seine mit Lucius Verus erworbenen Siegestitel auf offiziellen Dokumenten wegzulassen, zwar weitgehend umgesetzt wurde, auf den Papyri jedoch nicht befolgt ist.

Das zweite große Thema des Bandes („Rom und Germanien“, S. 61-108) ist das Verhältnis zwischen Rom und den germanischen Stämmen in den ersten vier Jahrhunderten der Kaiserzeit, das in drei Aufsätzen behandelt wird. Die Relevanz dieses Teilbereiches wird allerdings dadurch gemindert, dass Johne im Jahre 2006 eine größere und aktuellere Monographie über die Römer an der Elbe (S. 263) der Fachwelt vorgelegt hat, in die auch die Ergebnisse dieser Aufsätze eingegangen sind.

In dem zuerst in der Festschrift zum 75. Geburtstag von Karl Christ publizierten Aufsatz („Einst war sie ein hochberühmter und wohlbekannter Fluß“. Die Elbe in den Schriften des Tacitus“, S. 61-77) geht Johne von der Beobachtung aus, dass es sich bei der Elbe um den einzigen Fluss im Inneren Germaniens handelt, der von Tacitus erwähnt wird. Durch eine Analyse der entsprechenden Passagen kann er zeigen, dass Tacitus in der Germania der vergangenen Erfolge der augusteischen Zeit gedenkt, während die Nennungen in den Annales teils als Rückblick gedacht sind und teils das angestrebte Ziel des Germanicus darlegen sollen. Bei der Frage nach der Elbgrenze als Ziel der römischen Expansion spricht sich Johne für eine Entwicklung in vier Stufen aus, bei der spätestens im Jahre 9 n. Chr. die Elbe als Grenze des Reiches erstrebt, dies aber seit 17 n. Chr. trotz entgegenlautender Propagandistik faktisch aufgegeben wurde.

Ebenfalls ein Beitrag in einer Festschrift, diesmal für Achim Leube, ist „Semnonen am Lech. Der Augsburger Victoria-Altar und die Historia Augusta“ (S. 79-91). Der hier von Johne untersuchte Siegesaltar wurde 1992 in Augsburg entdeckt und nimmt auf Ereignisse der Germaneneinfälle in den Jahren 259 und 260 Bezug, die in der Historia Augusta (vermutlich aufgrund der Feindseligkeit des Biographen gegenüber Gallienus) unerwähnt bleiben. Deswegen und aufgrund der weitgehenden Unkenntnis der nachdionischen Autoren über die Verhältnisse im Inneren Germaniens bietet das Monument wichtige Ergänzungen zur literarischen Überlieferung.

Erneut mit der Elbe befasst sich „Die Elbe und der nördliche Ozean in der Historia Augusta“ (S. 93-108), ursprünglich ein Vortrag auf dem Kolloquium zur Historia Augusta in Perugia (2000) und im entsprechenden Kongressband publiziert. Auf Basis seiner Erkenntnisse zur Bedeutung der Elbe in den Schriften des Tacitus (S. 61-77, siehe dazu oben) untersucht Johne deren Bedeutung in der Historia Augusta, die mit diesem den einzigen außerhalb des römischen Reiches befindlichen Fluss nennt. Er gelangt zu dem Ergebnis, dass auch die Historia Augusta die Elbe und den nördlichen Ozean als angestrebtes Fernziel der römischen Expansionspolitik darstellen will und sich dieser Gedanke somit über die Jahrhunderte nach Augustus erhalten hat. 
Vier Aufsätze stellen den dritten thematischen Block („Studien zur Soldatenkaiserzeit“, S. 109-147), der sich mit der Zeit der Soldatenkaiser im dritten Jahrhundert befasst. Die Leitlinie aller hier abgedruckten Studien ist die Entwicklung des Kaisertums und die Wandlung der Voraussetzungen dafür vor dem Hintergrund der wachsenden Bedeutung der hohen militärischen Offiziere und der schwindenden des Senats sowohl als Reservoir für Kaiserkandidaten sowie als Institution zur Wahl oder auch nur Bestätigung neuer Kaiser.

Mit „Tacitus, der Kaiser und der Konsul“ (S. 109-116) wird eine für die soeben genannte Thematik wesentliche Persönlichkeit behandelt. Der nur wenige Monate in den Jahren 275 und 276 regierende Kaiser Tacitus gilt aufgrund seiner Herkunft und den Umständen seiner Wahl als letzter wirklicher Senatskaiser, nach dessen Herrschaft die Soldaten endgültig die alleinige Erhebung des Kaisers übernommen hätten. Der Beitrag behandelt gleichermaßen allgemeine Fragen (wie den Realitätsgehalt der genannten Annahme) und spezielle Probleme (vor allem das, ob der Kaiser Tacitus mit dem gleichnamigen Konsul des Jahres 273 identisch ist). Der Beitrag ist zweifellos noch immer lesenswert, wird aber mittlerweile durch den Beitrag Johnes im großen Handbuch zu den Soldatenkaisern (S. 267, siehe auch unten) überlagert.

Einen Vergleich des Kaisers Tacitus mit Oclatinius Adventus, einem der Prätorianerpräfekten des Kaisers Caracalla und, obwohl aus dem Soldatenstand stammend, nach dessen Tod als Nachfolger erwählt, zieht „Oclatinius Adventus und Claudius Tacitus zwei Karrieren aus der Soldatenkaiserzeit“ (S. 117-126). Die Vorgehensweise entspricht der des vorhergehenden Aufsatzes, so dass es dementsprechend starke Überschneidungen gibt. Durch den Vergleich und das Nachzeichnen der zwischenzeitlichen Entwicklungen kann Johne zeigen, wie sich in den Jahren von 217 (Tod Caracallas) bis 275 (Kaiserwahl des Tacitus) die Voraussetzungen für das Kaisertum gewandelt haben.

Bei dem Beitrag „Die illyrischen Kaiser als Herrscher neuen Typs“ (S. 127-138), ein Vortrag auf einer Tagung zu den Transformationsprozessen im römischen Reich des dritten Jahrhunderts (Humboldt-Universität Berlin, 8.-10. Juli 2005), handelt es sich um den jüngsten des Bandes. Johne betrachtet hierin erneut die Entwicklungen des römischen Kaisertums in den Jahren 217 bis letztlich 310 (erstmals Propagierung der angeblichen Abstammung Konstantins von Claudius II. Gothicus) mit Blick auf die soziale und lokale Herkunft der Kaiser und auf die wesentlichen Zäsuren (217 wird Macrinus als erster Ritter zum Kaiser, 235 Maximinus Thrax als erster Soldat, ab 268 Illyrer als Kaiser neuen Typs, 310 erneutes Hervortreten des dynastischen Elementes). Auch wenn der Aufsatz viele nützliche Einzelbeobachtungen bietet, kann er als einziger des Bandes nicht wirklich zufriedenstellen, da Johne sich größtenteils auf die Breviatoren des späteren vierten Jahrhunderts (insbesondere Aurelius Victor und seine Einteilung der Kaiserzeit) und somit auch nur auf im Rückblick urteilende Autoren stützt, während zeitgenössische Quellen weitgehend ausgeklammert bleiben. Als Beitrag zur Wahrnehmung der entsprechenden Entwicklungen der Reichskrise durch spätere Autoren kann man den Aufsatz allerdings als gelungen betrachten.

Auch bei „Das Offizierscorps des 3. Jahrhunderts als Reservoir einer neuen Führungsschicht" (S. 139-147) handelt es sich um einen Vortrag auf einem Kolloquium (Köln, 24.-26. November 1991), dessen Beiträge als Festschrift zum 80. Geburtstag von Leiva Petersen, der bereits erwähnten langjährigen Herausgeberin der PIR (S. 10), konzipiert waren. Wie die zuvor genannten Studien behandelt auch dieser Aufsatz denselben The- 
menkomplex, doch geht es nunmehr stärker um die Bedeutung des Soldatenstandes und der Offiziere im Allgemeinen, so wird etwa auch die Heeresreform des Gallienus einer genaueren Betrachtung unterzogen.

Der vierte thematische Block („Kolonen und Kolonat“, S. 149-196) behandelt mit dem Kolonat in römischer Zeit ein Themenfeld, das Johne erstmals in seiner Habilitationsschrift (1981, publiziert 1983) eingehender erforschte. Die vier Aufsätze sind zwar vor allem der Spätantike gewidmet, behandeln die Thematik allerdings aus unterschiedlichen Perspektiven und mit verschiedenen Schwerpunkten.

Eine exzellente Zusammenstellung ist die begriffsgeschichtliche Studie „Colonus, colonia, colonatus“ (S. 149-164), in der das Material zur Terminologie gesammelt und geordnet vorliegt und die Entwicklung der einzelnen Begriffe von der Republik bis in die Spätantike nachvollziehbar gemacht wird.

Mehr auf die archäologischen und epigraphischen Quellen konzentriert ist hingegen „Die Entwicklung von Kolonenwirtschaft und Kolonat mit besonderer Berücksichtigung der nördlichen Grenzprovinzen des Reiches“ (S. 165-179), ein Vortrag auf einem Kolloquium zur ländlichen Besiedlung und Landwirtschaft in den Rhein-Donau-Provinzen des römischen Reiches (Passau, 16.-21. April 1991). Der Wert des Beitrages besteht in der Diskussion der oft in ihrem genauen Wert für die Thematik unklaren archäologischen Zeugnisse und deren Kontextualisierung durch einen Vergleich mit den literarischen und juristischen Quellen.

Ebenfalls auf einen Vortrag geht der Beitrag „Zum Begriff Kolonat in der Spätantike“ (S. 181-185) zurück, der auf der 16. Internationalen Eirene-Konferenz (Prag, 31. August-4. September 1983) gehalten wurde. Der begriffsgeschichtlich interessierte Beitrag weist dieselben Vorzüge auf wie der bereits erwähnte fünf Jahre später erschienene (aber in diesem Band vor dem früheren Werk angeordnete) Aufsatz „Colonus, colonia, colonatus" (S. 149-164), geht jedoch nicht wesentlich über diesen hinaus, so dass sich daraus im Vergleich kein zusätzlicher Gewinn ergibt.

Einem sehr speziellen Thema gewidmet ist „Kolonen und Kolonat in der Historia Augusta“ (S. 187-196), ein Vortrag auf dem Bonner Historia-Augusta-Colloquium 1986/1989, worin die drei Erwähnungen von Kolonen in der Historia Augusta untersucht werden. Johne kann hierin zwar zeigen, dass alle drei Angaben unhistorisch sind, die ersten beiden jedoch mit der Evidenz in Einklang zu bringen sind und somit als historische Fakten theoretisch möglich wären. Da in der dritten Passage die Kolonen mit den Sklaven in einem Zug genannt werden, scheint das ihren Status der Halbfreiheit in der Spätantike vorauszusetzen und bietet somit einen vagen Hinweis auf die echte Abfassungszeit der Historia Augusta im späteren vierten Jahrhundert.

Mit dem fünften und letzten thematischen Block („Studien zur Historia Augusta“, S. 197-262) ist nach Ansicht des Rezensenten auch der Höhepunkt erreicht, da hier insgesamt die besten und wichtigsten unter den abgedruckten wie unter den Aufsätzen Johnes allgemein zu finden sind. Thema ist die bereits in einigen oben behandelten Beiträgen (S. 25-34; S. 79-91; S. 93-108; S. 187-196) thematisierte Historia Augusta, mit deren Problematik sich Johne seit seiner Dissertation (1971, publiziert 1976) befasst. Insgesamt sechs Aufsätze aus deren Umfeld sind hier erneut abgedruckt.

Der Aufsatz „Zum Geschichtsbild in der Historia Augusta“ (S. 197-209) ist in doppelter Hinsicht eine wertvolle Ergänzung zur Dissertation Johnes. Zum einen wird darin der 
exzellente Forschungsüberblick derselben für die Jahre 1889 bis etwa 1970, bei dem es sich bislang um den besten und ausführlichsten Überblick über das Datierungsproblem allgemein handelt, bis etwa 1982 fortgeführt. Zum anderen ergänzt Johne in diesem Aufsatz seine Untersuchungen zu den Elementen des in der Historia Augusta vorliegenden Geschichtsbildes der heidnischen stadtrömischen Senatsaristokratie; behandelt werden die Bedeutung Roms, die Beurteilung der Kaiser nach ihrem Verhältnis zum Senat sowie die Idealisierung der Republik und die Verklärung der Königszeit durch die Historia Augusta.

Die Abhandlung „Zur Widerspiegelung der Krise des römischen Reiches in der Historia Augusta“ (S. 211-218) stellt die Frage, ob sich die nach der Schlacht bei Adrianopel im Jahre 378 feststellbare Auflösung des Reiches in der Darstellung der Historia Augusta über die Außenpolitik der behandelten Kaiser nachweisen lässt. Nach Johnes Ansicht ist dies der Fall und der dort vertretene Eroberungsgedanke könne nur als Wunsch einer Zeit verstanden werden, in der die Germanen, nicht aber die Römer die Eroberer sind. Der Rezensent muss bekennen, in diesem Fall von der sich weitgehend auf Indizien und mögliche, aber nicht zwingende Schlussfolgerungen stützenden Beweisführung nicht überzeugt zu sein, doch macht bereits die eingehende Belegsammlung den Aufsatz zu einem wichtigen Hilfsmittel für die Erforschung der Historia Augusta.

Neben dem oben behandelten ersten (S. 53-59) ist auch der zweite jemals publizierte Aufsatz Johnes („Zur stadtrömischen Tendenz der Historia Augusta“, S. 219-226), erschienen im Themenband einer Zeitschrift über „Die antike Geschichtsschreibung und ihre gesellschaftlichen Grundlagen", in diesem Band enthalten. Hierin wird die stadtrömische Tendenz der Historia Augusta auf Basis der Beurteilung der Kaiser nach ihrem Verhältnis zum Senat und der in der Historia Augusta erfolgten Herabsetzung Konstantinopels, womit gegen die im Jahre 359 erfolgte Gleichstellung mit Rom protestiert werden sollte, herausgearbeitet. Der Aufsatz argumentiert überzeugend und ist ein relevanter Beitrag, allerdings wird sein Wert dadurch gemindert, dass die konkrete Thematik nur wenige Jahre später von Johne ausführlicher in seiner Dissertation behandelt wurde.

Gewissermaßen eine Fortsetzung und Ausweitung des letzten Aufsatzes ist der Beitrag zu „Byzanz in der Historia Augusta“ (S. 227-239). Johne demonstriert hier, dass die Historia Augusta zwei Formen der Erwähnungen von Byzanz bietet. Die Angaben über die Bestrafung durch Septimius Severus nach dem Bürgerkrieg gegen Pescennius Niger sowie über den Aufenthalt Aurelians in dieser Stadt und seine Ermordung in deren Nähe sind historisch und somit durch die vorhandenen Tatsachen bestimmt. Die Behauptung hingegen, Soldaten des Gallienus hätten die Stadt verwüstet und keine Person aus dem lokalen Adel am Leben gelassen, zielt auf eine Herabsetzung der Stadt ab, was durch die Gleichstellung mit Rom im Jahre 359 zu erklären ist. Die sich daraus ergebende Datierung der Historia Augusta kann Johne durch einen Vergleich mit einer Reihe von Kritikern Konstantinopels aus dem späteren vierten Jahrhundert erhärten.

Der Aufsatz über „Die Rolle der Kaiserresidenzen in der Historia Augusta“ (S. 241-250) geht dasselbe Thema in etwas allgemeinerer Form an, indem neben Byzanz auch die übrigen spätantiken Kaiserresidenzen und ihre Darstellung in der Historia Augusta betrachtet werden. Eher negativ beurteilt werden Byzanz, Antiochia, Sirmium und Mediolanum, weitgehend unberücksichtigt sind Nikomedia (das nach der Aufwertung Konstantinopels deutlich an Bedeutung verloren hatte), Trier und Thessalonike, 
Serdica bleibt vollkommen unerwähnt. Entsprechend zeigt sich eine allgemeine Tendenz zur Herabsetzung der spätantiken Hofstädte. Sehr interessant ist auch die Beobachtung Johnes, dass die neutrale Darstellung des nur in historisch gesicherten Zusammenhängen genannten Ravenna darauf hindeutet, dass diese Stadt noch nicht den Status einer Kaiserresidenz hatte und die Historia Augusta somit wahrscheinlich vor dem Jahr 404 abgefasst wurde. Auch dieser Aufsatz ist verdienstvoll und nützlich, doch droht das Publikationsjahr (1978) in die Irre zu führen: Da er anscheinend zu einer Zeit verfasst wurde, als Johne zwar bereits seine Dissertation fertiggestellt hatte (1971/1972), deren Buchfassung (1976) jedoch noch nicht publiziert oder abgeschlossen war (S. 243, Anm. 3 = S. 355, Anm. 3 der Erstpublikation, wo nur die frühere Fassung erwähnt ist), liegt somit mit dem entsprechenden Kapitel ebenjener Buchfassung (V, 2: S. 156-176) eine ausführlichere und aktuellere Version des Aufsatzes vor.

Einer speziellen Vita der Historia Augusta gewidmet ist „Die Biographie des Gegenkaisers Censorinus. Ein Beitrag zur sozialen Herkunft der Historia Augusta“ (S. 251-262), ursprünglich ein Vortrag auf dem Bonner Historia-Augusta-Colloquium 1972/1974, worin die Lebensbeschreibung des vollständig erfundenen Usurpators Censorinus, der sich gegen Claudius II. Gothicus erhoben haben soll, geprüft wird. Johne kann hier zeigen, wie diese Vita aus literarischen Anspielungen und Elementen anderer Viten der Historia Augusta zusammengesetzt ist, in der fiktiven Laufbahn des Censorinus Elemente des cursus des 2./3. Jahrhunderts mit dem des 4./5. Jahrhunderts vermengt sind (so setzt der Aufstieg vom Stadtpräfekten zum Prätorianerpräfekten die konstantinischen Reformen voraus) und sich die Verbindungen des Autors zu den stadtrömischen Aristokratenfamilien der Spätantike auch in dieser Vita erkennen lassen (etwa in der Bedeutung der Stadtpräfektur). Der gelungene Aufsatz ist im selben Jahr auch als Teil eines Kapitels in der Buchfassung der Dissertation Johnes erschienen (IV, 2: S. 121-129).

Soweit zu den Inhalten des Bandes (auf das Schriftenverzeichnis Johnes wird hingegen unten noch ausführlicher eingegangen). Nun ist die Frage zu stellen, welchen Wert er als Gesamtwerk hat.

Auf der einen Seite gibt es viel Gutes zu sagen: Die Qualität der einzelnen Aufsätze ist exzellent, viele sind zudem noch immer aktuell und wichtig, einige nur schwer zugängliche Publikationen Johnes sind auf diesem Wege leicht zugänglich, das (fast) vollständige Schriftenverzeichnis ist als bibliographisches Hilfsmittel wertvoll und der Band bietet einen guten Querschnitt durch das Gesamtwerk Johnes. Zudem nützt der Band nicht nur dem eigentlichen Altertumswissenschaftler, sondern auch für den Wissenschaftshistoriker lohnt sich ein Blick, da einerseits die ersten beiden Aufsätze des Bandes (S. 17-24; S. 25-34) die Geschichte der PIR behandeln und andererseits Person und Werk Johnes für einen Erforscher der Geschichte der Altertumswissenschaften in der DDR von Interesse sein können (für einige Ansätze dazu siehe unten).

Bei allen Verdiensten weist der Band jedoch auch verschiedene (teils grundlegende) Schwächen auf. Ich behandle die einzelnen Aspekte in aufsteigender Reihenfolge nach der ungefähren Schwere ihrer Problematik.

Druckfehler und Ähnliches treten immer wieder auf, haben jedoch fast nie größere Auswirkungen. Manchmal wurde versäumt, Fehler, die bereits in der Erstpublikation 
(EP) auftauchen, zu korrigieren; ${ }^{4}$ etwas größer ist die Zahl der erst durch die Neupublikation eingegangenen Fehler. ${ }^{5}$

Einen doppelten Anlass zur Kritik bietet der Umgang mit den Querverweisen. Das erste Problem ist die Tatsache, dass die Erstpublikationen an den unterschiedlichsten Orten erschienen sind und somit auch die Standards bei den Querverweisen innerhalb der Aufsätze abweichen: Während in vielen Aufsätzen Vollzitate durch eindeutige Verweise auf frühere Anmerkungen schnell und einfach gefunden werden können und in zwei Fällen ein Verzeichnis am Ende des jeweiligen Aufsatzes die durch Kurztitel angegebenen Literaturzitate auflöst (S. 89-91; S. 137-138), finden sich manchmal Fälle, in denen Querverweise nicht oder nur sehr unsystematisch auftauchen (unsystematisch: S. 149-164; keine: S. 139-147; S. 219-226; S. 241-250; S. 251-262).

Der zweite Kritikpunkt zu den Querverweisen betrifft die Tatsache, dass es, obwohl die Aufsätze in neuer Setzung gedruckt sind und sich entsprechende Ergänzungen somit leicht hätten durchführen lassen, versäumt wurde, auf in demselben Band ebenfalls erneut abgedruckte Aufsätze entsprechend zu verweisen. ${ }^{6}$ Dass dies grundsätzlich möglich gewesen wäre, bezeugt S. 232, Anm. 22, wo ein Querverweis innerhalb dieses Aufsatzes gegenüber der (übrigens fehlerhaften: EP S. 126, Anm. 22) Vorlage so verändert wurde, dass er auch in der Neupublikation nachvollzogen werden kann; auch wurde S. 138 ein kurzes zusätzliches Literaturverzeichnis für die Fälle ergänzt, in denen sich der Aufsatz auf einen anderen Beitrag des Bandes, in dem er zuerst publiziert wurde, bezieht und die Anmerkungen sind entsprechend ergänzt (S. 129, Anm. 7; S. 130, Anm. 11; S. 131, Anm. 16; S. 134, Anm. 26; S. 136, Anm. 36).

4 Besonders gravierend ist die Tatsache, dass S. 23, Anm. 21 = EP S. 25, Anm. 21 zwei falsche bibliographische Angaben erhalten geblieben sind, da die zitierten Beiträge nicht in Revue des études latines 49, 1971 und in Epigraphica 33, 1971, sondern in den Bänden 48, 1970 und 32, 1970 erschienen sind. S. 127 = EP S. 125 fehlt ein Komma („Geta erst“, richtig „Geta, erst"); S. 132 = EP S. 129 ist „letzten“ statt „letzen“ zu lesen; S. 251, Anm. 1 = EP S. 131, Anm. 1 ist die alphabetische Reihenfolge nicht ganz eingehalten. Nicht falsch, aber ungewöhnlich sind S. 19 = EP S. 23 "Quelle“ statt "Quellen" (mit Blick auf den Kontext des Satzes); S. 101, Anm. 42 = EP S. 300, Anm. 42 „Julian. conv.“ (üblicher ist „Caes.“) und die Bezeichnung des Jahres 69 als „Dreikaiserjahr" (S. 128 = EP S. 126).

5 S. 18 wird durch fehlerhafte Kleinschreibung die Satzstruktur durcheinandergebracht („u. Z. alle“ statt EP S. 22 „u. Z. Alle“); S. 54 „nämlich, die“ (EP S. 178 „nämlich die“); S. 57, Anm. 25 ,in. der“ (EP S. 180, Anm. 4 ,in der“); S. 57 wäre Sarmaticus noch kursiv zu setzen (richtig EP S. 180); S. 58 sind an einer Stelle die griechischen Buchstaben (EP S. 181) durch lateinische (zudem mit falscher Transkription) ersetzt; S. 85, Anm. 20 „Quellen in“ (EP S. 302, Anm. 20 „Quellen III“); S. 124 „Pertinax. war“; S. 141 „Maximums“ (EP S. 253 „Maximinus“); S. 145 „proviorischen“ (EP S. 256-257 "provisorischen“); S. 161 „Jahrs" (EP S. 319 „Jahre“); S. 213-217 jeweils in der Titelzeile „Reichesin“ statt richtig „Reiches in“. Recht häufig sind fehlende oder zusätzliche Leerzeichen (S. 35; S. 75, Anm. 59; S. 86, Anm. 25; S. 123, Anm. 20; S. 237, Anm. 48; S. 261, Anm. 44) und vor allem sonderbare Worttrennungen (S. 32; S. 82, Anm. 8; S. 83; S. 95, Anm. 7; S. 96; S. 98; S. 110; S. 115-116; S. 122; S. 145; S. 199; S. 215; S. 223; S. 233; S. 242; S. 248).

6 Ich stelle die Fälle zusammen, in denen Querverweise zu ergänzen wären (die Zahl vor den Klammern ist die Stelle, wo der Verweis einzufügen wäre, die in Klammern bezeichnet die Seiten, auf die zu verweisen ist): S. 27, Anm. 6 (S. 17-24); S. 35, Anm. 2 (S. 17-24); S. 85, Anm. 20 und S. 90 (S. 61-77); S. 94, Anm. 6 (S. 61-77; S. 68-69); S. 95, Anm. 7 (S. 72-76); S. 96, Anm. 11 (S. 64-66; S. 75-77); S. 103, Anm. 52 (S. 211-218); S. 109, Anm. 1 (S. 197-209); S. 124, Anm. 26 (S. 109-116); S. 125, Anm. 32 (S. 110, Anm. 4; S. 112, Anm. 12); S. 133, Anm. 24 und S. 137 (S. 117-126); S. 194, Anm. 27 (S. 149-164); S. 205, Anm. 36 (S. 251-262); S. 231, Anm. 17 (S. 251-253). Übersehen wurde auch ein Querverweis innerhalb eines Aufsatzes, da S. 162 noch auf "Seite 309“ (richtig wäre S. 150-151) verwiesen wird, was allerdings durch die durchgehend gebotene parallele Angabe der Paginierung der Erstpublikation zumindest leichter nachzuvollziehen ist. 
Es wurde darauf verzichtet, die Aufsätze zu überarbeiten und eine Auseinandersetzung mit der neueren Literatur zu bieten. Das ist grundsätzlich nachvollziehbar, doch wären zwei Ergänzungen zweifellos nützlich gewesen. Zum einen hätte man am Schluss der einzelnen Aufsätze oder des Bandes eine kurze Liste mit der zwischenzeitlich erschienenen Spezialliteratur zu dem jeweiligen Aufsatzthema bieten können. Zweitens wäre es gut möglich gewesen, wenn denn von Eingriffen in den eigentlichen Inhalt der Aufsätze abgesehen werden soll, zumindest auf Zweitpublikationen ${ }^{7}$ und Neuauflagen ${ }^{8}$ (sowie in einigen Fällen auf Erstpublikationen) von zitierten Werken zu verweisen.

7 Folgende Ergänzungen wären hier möglich gewesen: Géza Alföldy, Die römische Gesellschaft, Stuttgart: Franz Steiner, 1986, S. 82-98 (zu S. 143, Anm. 16) und S. 434-484 (zu S. 187, Anm. 1; S. 200, Anm. 8; S. 201, Anm. 15; S. 202, Anm. 25); Géza Alföldy, Die Krise des römischen Reiches, Stuttgart: Franz Steiner, 1989, S. 25-68 (zu S. 215, Anm. 15); S. 390-405 (zu S. 225, Anm. 34) und S. 406-418 (zu S. 200, Anm. 8); Barry Baldwin, Studies on late Roman and Byzantine history, literature and language, Amsterdam: J. C. Gieben, 1984, S. 51 (zu S. 200, Anm. 8); Barry Baldwin, Roman and Byzantine papers, Amsterdam: J. C. Gieben, 1989, S. 197-200 (zu S. 200, Anm. 8); Timothy David Barnes, Early Christianity and the Roman empire, London: Variorum Reprints, 1984, Nr. IV (zu S. 111, Anm. 9); Eric Birley, The Roman army, Amsterdam: J. C. Gieben, 1988, S. 44-52 (zu S. 200, Anm. 11); Jochen Bleicken, Gesammelte Schriften II, Stuttgart: Franz Steiner, 1998, S. 901-953 (zu S. 225, Anm. 37); André Chastagnol, Aspects de l'antiquité tardive, Rom: L'Erma di Bretschneider, 1994, S. 259-274 (zu S. 108, Anm. 70); André Chastagnol, Le pouvoir impérial à Rome, Genf: Droz, 2008, S. 29-55 (zu S. 202, Anm. 23); Alexander Demandt, Zeitenwende, Berlin: Walter De Gruyter, 2013, S. 52-84 (zu S. 137; S. 147, Anm. 32) und S. 112-133 (zu S. 206, Anm. 44); Ernst Hohl (Übs.), Historia Augusta I, Zürich: Artemis-Verlag, 1976, S. 1-27 und S. 371-373 (beides zu S. 25, Anm. 2); Adolf Lippold, Die Historia Augusta, Stuttgart: Franz Steiner, 1998, S. 15-33 (zu S. 30, Anm. 16); S. $82-96$ (zu S. 139, Anm. 4; S. 204, Anm. 33); S. 97-113 (zu S. 32, Anm. 26-27); S. 114-130 (zu S. 103, Anm. 52; S. 106-107, Anm. 64) und S. 213-229 (zu S. 217, Anm. 23); Arnaldo Momigliano, Ausgewählte Schriften zur Geschichte und Geschichtsschreibung I, Stuttgart: J. B. Metzler, 1998, S. 313-349 und S. 407-416 (beides zu S. 219, Anm. 3 und S. 233, Anm. 31, deren Angaben über die weiteren Publikationsorte sich gegenseitig ergänzen); Theodor Mommsen, Gesammelte Schriften VII, Berlin: Weidmannsche Buchhandlung, 1909, S. 298-301 (zu S. 257, Anm. 20); Alfons Rösger, Studien zum Herrscherbegriff der Historia Augusta und zum antiken Erziehungswesen, Frankfurt a. M.: Peter Lang, 2001, S. 11-64 (zu S. 200, Anm. 11; S. 204, Anm. 35); Wolfgang Schmid, Ausgewählte philologische Schriften, Berlin: Walter de Gruyter, 1984, S. 616-625 (zu S. 199, Anm. 3; S. 223, Anm. 22; S. 227, Anm. 1); William Seston, Scripta varia, Paris: École française de Rome, 1980, S. 109-117 und S. 215-219 (beides zu S. 45, Anm. 2); Johannes Straub, Regeneratio imperii, Darmstadt: Wissenschaftliche Buchgesellschaft, 1972, S. 329-349 (zu S. 32, Anm. 25) und S. 410-417 (zu S. 226, Anm. 39); Ronald Syme, Emperors and biography, Oxford: Clarendon Press, 1971, S. 1-16 (zu S. 238, Anm. 49); Ronald Syme, Roman papers I, Oxford: Clarendon Press, 1979, S. 271-291 (zu S. 42, Anm. 31); Ronald Syme, Historia Augusta papers, Oxford: Clarendon Press, 1983, S. 109-130 (zu S. 199-205, dort vierzehn Zitate der Erstpublikation); Dieter Timpe, Römisch-germanische Begegnung in der späten Republik und frühen Kaiserzeit, München: K. G. Saur, 2006, S. 147-170 (zu S. 73, Anm. 48; S. 95, Anm. 8); S. 171-190 (zu S. 71, Anm. 41); S. 191-215 (zu S. 67, Anm. 30; S. 93, Anm. 3) und S. 358-399 (zu S. 65, Anm. 19); Karl-Wilhelm Welwei, Res publica und Imperium, Wiesbaden: Franz Steiner, 2004, S. 275-280 (zu S. 178, Anm. 42); Richard Whittaker, Land, city and trade in the Roman empire, Aldershot: Variorum, 1993, Nr. I (zu S. 152, Anm. 13; S. 189, Anm. 7) und Nr. IV (zu S. 178, Anm. 42; S. 193, Anm. 22).

8 Die Dissertation von Ernst Hohl wird stets nach der (nicht ganz einfach zugänglichen) monographischen Fassung zitiert (S. 109, Anm. 3; S. 195, Anm. 28; S. 204, Anm. 34), während der damit identische, aber leichter zugängliche Zeitschriftenaufsatz Klio 11, 1911, S. 178-229 und S. 284-324 nie angeführt wird. Dietmar Kienasts Kaisertabelle wird meist nach der ersten Auflage zitiert (S. 97, Anm. 15; S. 101, Anm. 43; S. 120, Anm. 12; S. 123, Anm. 21; S. 124, Anm. 28; S. 125, Anm. 29), während die wesentlich überarbeitete und ergänzte zweite Auflage erst zehn Jahre nach ihrer Publikation Berücksichtigung gefunden hat (S. 137); auch Lippolds Biographie des Theodosius I. (S. 215, Anm. 13) und Birleys Biographie des Septimius Severus (S. 228, Anm. 6) liegen in erweiterten Neuauflagen vor. Die in S. 196, Anm. 33 gebotenen Angaben zu Jones können aus den vollständigeren anderer Aufsätze (S. 149, Anm. 1; S. 165-166, Anm. 1; S. 182, Anm. 1) ergänzt werden. Die Orte von Erstpublikationen lassen sich aus den zitierten Werken entnehmen (S. 57, Anm. 29; S. 143, Anm. 16; S. 145, Anm. 25; 
Zu der Sammlung der Liste von Rezensionen zu den Faszikeln IV, 3 und V, 1 der PIR (S. 23, Anm. 21) ließen sich noch vier hinzufügen: Zu IV, 3 die von Edmond Van't Dack, Revue belge de philologie et d'histoire 48, 1970, S. 577-578 und Ladislav Vidman, Listy filologické 93, 1970, S. 68-69 sowie zu V, 1 die von Malcolm A. R. Colledge, Classical Review $86=$ N. S. 22, 1972, S. 284 und Sergio Daris, Aegyptus 55, 1975, S. 308-309.

Vielleicht mag es erlaubt sein, an dieser Stelle zwei kurze Bemerkungen zu einzelnen Thesen der Aufsätze einzufügen, die zu weiteren Forschungen anregen könnten:

Mit Blick auf die Quellenverhältnisse der Historia Augusta stellt Johne fest „die Abhängigkeit von Aurelius Victor und Eutrop [ist] allgemein anerkannt" (S. 199 = EP S. 633, ähnlich S. 212 = EP S. 618). Das trifft für Aurelius Victor (mit Ausnahme der wenigen Forscher, die eine vollkommen vom üblichen Konsens abweichende Datierung der Historia Augusta vertreten) in der Tat zu, nicht aber für Eutropius, da die Frage, ob dessen Werk von der Historia Augusta direkt benutzt wurde oder ob beide auf eine gemeinsame Quelle zurückgegriffen haben, noch immer nicht mit letzter Sicherheit beantwortet ist.

Laut Johnes Aussage (S. 136 = EP S. 133) ist Aurelius Victor der einzige Autor, der den Kaiser Claudius II. Gothicus (268-270) nicht an der Pest sterben lässt, sondern für ihn einen heroischen Opfertod im Dienste des Staates nach dem Vorbild der republikanischen Senatorenfamilie der Decier, von denen drei Vertretern ein solcher Tod zugeschrieben wird, angibt. Tatsächlich spricht aber auch Ammianus Marcellinus (Amm. XVI, 10, 3; ergänzend Amm. XXXI, 5, 17; siehe auch Epitome de Caesaribus 34, 3-4) von einem Kaiser, der wie die Decii sein Leben für den Staat gab. Dieser Kaiser wird von ihm nicht namentlich genannt, doch besteht in den Kommentaren und Diskussionen dieser Stelle Einigkeit darüber, dass es sich um Claudius II. Gothicus handelt. Da Aurelius Victor ein überzeugter Senator und Zivilist mit Abneigung gegen das Heer war, Ammianus sich hingegen offen als ehemaliger Soldat darstellt, bieten diese beiden Passagen einen interessanten Ansatzpunkt für eine Untersuchung der Geisteswelt des vierten Jahrhunderts und ihrer Beeinflussung durch die Wandlungen im dritten Jahrhundert.

Die entscheidende Frage ist nun die nach dem Band als Gesamtwerk. Warum stellt man (von persönlichen Gründen wie der Ehrung eines Forschers abgesehen) einen Band Kleiner Schriften zusammen? Insgesamt sind dafür vier mögliche Gründe zu nennen: 1) Das Gesamtwerk eines Forschers soll entweder vollständig oder in seinen wesentlichen Elementen abgebildet werden. 2) Eine Reihe wichtiger Aufsätze zu einem bestimmten Thema soll so gesammelt vorliegen. 3) An abgelegenen Orten publizierte Aufsätze sollen auf diesem Weg leichter zugänglich gemacht werden. 4) Durch die bei einem Einzelwerk bestehenden Möglichkeiten (etwa ein ausführlicher Registerteil) sollen so die Aufsätze besser erschlossen werden.

Die Abbildung von Johnes Gesamtwerk ist größtenteils gelungen und die wesentlichen Stationen seiner Forschungstätigkeit sind gut repräsentiert. Den Gesamteindruck abgerundet hätten allerdings einige seiner größeren und wichtigeren Beiträge für die PIR (siehe dazu auch die Liste unten) und die im nächsten Absatz angeführten Werke.

Bedingt gelungen ist die Sammlung wichtiger Aufsätze zu einem bestimmten Thema. Während die wichtigsten Beiträge Johnes zur Historia Augusta in diesem Band

S. 201, Anm. 18; S. 203, Anm. 32; S. 237, Anm. 45), weswegen sie hier nicht im Einzelnen angeführt sind. 
versammelt sind, haben die Auswahl der Aufsätze bei den anderen Themenkomplexen und deren häufige Überschneidung untereinander immer wieder zur Folge, dass manche Themenfelder zugunsten anderer überrepräsentiert sind. So haben die Studien zur Soldatenkaiserzeit (S. 109-147) insgesamt schwerpunktmäßig den Kaiser Tacitus zum Thema, während Johnes nicht weniger interessante Studie zu Maximinus Thrax in der Festschrift für Jan Burian (S. 265) nicht aufgenommen ist. Ebenso sind die Beiträge zum Kolonat (S. 149-196) sehr stark auf die Spätantike fixiert, was aber zumindest dadurch ausgeglichen wird, dass die frühere Zeit eingehend in der Buchfassung von Johnes Habilitationsschrift (S. 263) behandelt ist. Umgekehrt widmen sich die Beiträge zur Prosopographie (S. 17-59) vor allem den ersten drei Jahrhunderten der Kaiserzeit, während der in der Festschrift für Günther Christian Hansen publizierte Beitrag zur Prosopographie der Spätantike (S. 266) nicht in den Band aufgenommen wurde.

Ebenfalls bedingt erfolgreich ist die Zugänglichmachung abgelegen publizierter Aufsätze. Da einige Beiträge in Festschriften oder wenig bekannten Zeitschriften erschienen sind, werden diese vermutlich erst durch die Neupublikation überhaupt einem größeren Fachpublikum zugänglich. Bei den meisten Sammlungen wäre es natürlich wenig sinnvoll, diesen Aspekt an erster Stelle zu setzen, aber in manchen Fällen hätte eine ausgewogenere Auswahl mit Blick auf den vorhergehenden Aspekt auch den Wert des Bandes in dieser Hinsicht erhöht; so etwa im Fall des oben erwähnten Festschriftenaufsatzes zu Maximinus Thrax (S. 265).

Im vierten Punkt versagt der Band leider fast vollkommen. Das Fehlen von Aktualisierungen und Querverweisen wurde bereits erwähnt. Ebenfalls versäumt wurde die Anfertigung eines Registers, so dass die (manchmal sehr begrenzten) Registerteile der Erstpublikationsorte sowie die mittlerweile teilweise als Digitalisate (mit entsprechenden Suchfunktionen) vorliegenden Zeitschriftenaufsätze hier von größerem Nutzen sind.

Bevor nun zu einem Gesamturteil gelangt werden kann, ist noch auf das Schriftenverzeichnis Johnes am Schluss des Bandes (S. 263-270) einzugehen. Dieses ist einer der wertvollsten Teile des Bandes, da es eine in Schriftenverzeichnissen selten gewordene Vollständigkeit aufweist. Erfasst sind nämlich auch kleinere Aufsätze sowie nahezu die gesamte Rezensionstätigkeit Johnes. Als bibliographisches Instrument hat das Verzeichnis einen großen Wert und es bedarf eingehender Recherchen, um die wenigen bestehenden Fehlstellen auszumachen.

Insgesamt konnten genau vierzehn Publikationen Johnes aus dem von dem Schriftenverzeichnis abgedeckten Zeitraum ermittelt werden, die darin nicht berücksichtigt sind. Dabei handelt es sich um die drei ursprünglichen Qualifikationsschriften (Nr. 1-2, 4), zwei Beiträge anlässlich von Geburtstagen seines akademischen Lehrers Wolfgang Seyfarth (Nr. 3, 5), ${ }^{9}$ ein Bericht über ein Kolloquium zu Ehren von Werner Hartke (Nr. 6) dessen Ergebnisse im selben Jahr publiziert wurden (S. 265) -, drei allgemeinere Beiträge in ansonsten vollständig erfassten Sammelbänden (Nr. 8, 12, 14), drei Rezensionen (Nr. 9-11), einen Beitrag in einem Kongressband (Nr. 7) und ein Vorwort zur Buchfassung einer von ihm betreuten Magisterarbeit (Nr. 13).

9 Der Nachruf auf ihn wurde dann aber von zwei anderen Schülerinnen Seyfarths verfasst: Liselotte Jacob-Karau, Ilse Ulmann, „Wolfgang Seyfarth zum Gedenken“, Ethnographisch-archäologische Zeitschrift 27, 1986, S. 278-280. 
1) Diplomexamensarbeit mit nicht ermittelbarem Titel und Thema (Historia Augusta?) [siehe nur kurz und sehr allgemein S. 9].

2) Untersuchungen zur Datierung und sozialen Herkunft der Historia Augusta, Diss., Berlin, Humboldt-Universität, 1971 [ein Inhaltsverzeichnis findet sich im Jahrbuch für Wirtschaftsgeschichte 1971/3, S. 296].

3) „Wolfgang Seyfarth zum 70. Geburtstag“, Ethnographisch-archäologische Zeitschrift 17, 1976, S. 109-110.

4) Die Kolonen in Italien und den westlichen Provinzen des Römischen Reiches vom 2.Jahrhundert v.u.Z. bis zu den Severern nach den literarischen Quellen, Diss. B [Habil.-Schr.], Berlin, Akademie der Wissenschaften der DDR, 1981 [die Zusammenfassung in der Ethnographisch-archäologischen Zeitschrift ist S. 265 angeführt].

5) „Wolfgang Seyfarth 75 Jahre“, Zeitschrift für Geschichtswissenschaft 29, 1981, S. 623.

6) „Rom und Germanien. Kolloquium zu Ehren von Akademiemitglied Werner Hartke, Berlin 1982“, Ethnographisch-archäologische Zeitschrift 24, 1983, S. 164-165.

7) „Kolonenwirtschaft und Kolonat“, in: XVe conférence internationale d'études classiques Eirene, Bukarest: Ed. Acad. Republicii Socialiste România / Sofia: Balgarska akademija na naukite, 1985, S. 66-71.

8) „Zusammenfassung der Ergebnisse“, in: Klaus-Peter Johne (Hrsg.), Gesellschaft und Wirtschaft des Römischen Reiches im 3. Jahrhundert, Berlin: Akademie-Verlag, 1993, S. 377-384.

9) Zeitschrift für Geschichtswissenschaft 44, 1996, S. 841-843 [zu Gerhard Fink, Who's who in der antiken Mythologie, München: Deutscher Taschenbuch-Verlag, 1993].

10) Altertum 43, 1997, S. 69-70 [zu Auguste V. B. Miron, Andrei Miron, Argonautika, Stuttgart: Konrad Theiss, 1995].

11) Altertum 43, 1997, S. 73-74 [zu Christian Leiber (Hrsg.), Schätze der Ostgoten, Stuttgart: Konrad Theiss, 1995].

12) Mit Thomas Gerhardt und Udo Hartmann, „Einleitung“, in: Klaus-Peter Johne, Thomas Gerhardt, Udo Hartmann (Hrsg.), Deleto paene imperio Romano, Stuttgart: Franz Steiner, 2006, S. 7-10.

13) „Vorwort“, in: Katja Wannack, Hermann Dessau, Hamburg: Dr. Kovač, 2007 (Magisterarbeit Berlin, Humboldt-Universität 2004), S. IX-X.

14) Mit Udo Hartmann, „Krise und Transformation des Reiches im 3. Jahrhundert“, in: Klaus-Peter Johne (Hrsg.), Die Zeit der Soldatenkaiser II, Berlin: Akademie-Verlag, 2008, S. 1025-1053.

Das Schriftenverzeichnis enthält S. 263, S. 266-267 und S. 270 neun Titel, die zum Zeitpunkt des Drucks abgefasst, aber noch nicht publiziert waren. Diese sind hier nachgetragen.

1) H-Soz-Kult 30. Juli 2007 = Historische Literatur 5/3, 2007, S. 17-19 [zu Peter Eich, Zur Metamorphose des politischen Systems in der römischen Kaiserzeit, Berlin: Akademie-Verlag, 2005].

(https://www.hsozkult.de/review/id/reb-7345)

(https://edoc.hu-berlin.de/bitstream/handle/18452/18500/HistLit_2007-3.pdf)

2) $H$-Soz-Kult 21. August $2007=$ Historische Literatur 5/3, 2007, S. 48-51 [zu Gerhard Rasch, Antike geographische Namen nördlich der Alpen, Berlin: Walter De Gruyter, 2005]. 
(https://www.hsozkult.de/review/id/reb-7341)

(https://edoc.hu-berlin.de/bitstream/handle/18452/18500/HistLit_2007-3.pdf)

3) „Zum Begriff Germania in der Historia Augusta“, in: Giorgio Bonamente, Hartwin Brandt (Hrsg.), Historiae Augustae Colloquium Bambergense, Bari: Edipuglia, 2007, S. 245-258.

4) Herausgeber von: Die Zeit der Soldatenkaiser I-II, Berlin: Akademie-Verlag, 2008.

5) Mit Thomas Gerhardt und Udo Hartmann, „Einleitung“, in: Die Zeit der Soldatenkaiser I, Berlin: Akademie-Verlag, 2008, S. 5-12.

6) „Die Historia Augusta“, in: Die Zeit der Soldatenkaiser I, Berlin: Akademie-Verlag, 2008, S. 45-51.

7) „Der „Senatskaiser“ Tacitus“, in: Die Zeit der Soldatenkaiser I, Berlin: Akademie-Verlag, 2008, S. 379-393.

8) „Das Kaisertum und die Herrscherwechsel“, in: Die Zeit der Soldatenkaiser I, Berlin: Akademie-Verlag, 2008, S. 583-632.

9) „Hermann Dessau und die Anfänge der prosopographischen Forschung“, in: Manfred G. Schmidt (Hrsg.), Hermann Dessau (1856-1931), Berlin: Walter de Gruyter, 2009, S. 31-46.

Da die mit diesem Beitrag verbundenen Recherchen auch Material zu einer Fortsetzung des Schriftenverzeichnisses erbracht haben, ${ }^{10}$ seien der Fachwelt deren Ergebnisse nicht vorenthalten. Ermittelt wurden sechs Aufsätze und zwanzig Rezensionen.

Die Aufsätze:

1) „Das Gallische Sonderreich“, in: Alexander Demandt, Josef Engemann (Hrsg.), Imperator Caesar Flavius Constantinus. Konstantin der Grosse, Mainz: Philipp von Zabern, 2007, S. 48-50.

2) „Die Langobarden in den Schriftquellen bis zu den Markomannenkriegen“, in: Jan Bemmann, Michael Schmauder (Hrsg.), Kulturwandel in Mitteleuropa, Bonn: Rudolf Habelt, 2008, S. 43-50.

3) "'Schon ist die Elbe näher als der Rhein“. Zur Diskussion um die Elbgrenze des Imperium Romanum“, Gymnasium 115, 2008, S. 237-250.

4) „Krisenwahrnehmung in der Historia Augusta“, in: Andreas Goltz, Hartmut Leppin, Heinrich Schlange-Schöningen (Hrsg.), Jenseits der Grenzen. Beiträge zur spätantiken und frühmittelalterlichen Geschichtsschreibung, Berlin: Walter De Gruyter, 2009, S. 79-90.

5) „Das Stromgebiet der Elbe im Spiegel der griechisch-römischen Literatur“, in: Ernst Baltrusch u.a. (Hrsg.), 2000 Jahre Varusschlacht, Berlin: Walter De Gruyter, 2012, S. 25-58.

6) „Klienten, Klientelstaaten und Klientelkönige bei den Germanen“, in: Ernst Baltrusch, Julia Wilker (Hrsg.), Amici - socii - clientes? Abhängige Herrschaft im Imperium Romanum, Berlin: Edition Topoi, 2015, S. 225-242.

Die Rezensionen:

1) H-Soz-Kult 21. Januar 2008 = Historische Literatur 6/1, 2008, S. 26-29 [zu Olivier Hekster u.a. (Hrsg.), Crises and the Roman empire, Leiden: Brill, 2007].

10 Ebenfalls ergab sich im Rahmen dessen (durch den gleichen Nachnamen) ein vielleicht nicht vollständiges, aber doch zumindest alle wesentlichen Beiträge von Renate Johne erfassendes Verzeichnis. Darüber ist jedoch an anderer Stelle zu handeln. 
(https://www.hsozkult.de/review/id/reb-10768)

(https://edoc.hu-berlin.de/bitstream/handle/18452/18515/HistLit_2008-1.pdf)

2) Klio 90, 2008, S. 511-512 [zu Jochen Haas, Die Umweltkrise des 3. Jahrhunderts n. Chr. im Nordwesten des Imperium Romanum, Stuttgart: Franz Steiner, 2006].

3) H-Soz-Kult 16. März 2009 = Historische Literatur 7/1, 2009, S. 41-42 [zu Ralf-Peter Märtin, Die Varusschlacht, Frankfurt a. M.: S. Fischer, 2008].

(https://www.hsozkult.de/review/id/reb-12147)

(https://edoc.hu-berlin.de/bitstream/handle/18452/18503/HistLit_2009-1.pdf)

4) H-Soz-Kult 21. September $2009=$ Historische Literatur 7/3, 2009, S. 82-83 [zu Helmuth Schneider (Hrsg.), Feindliche Nachbarn. Rom und die Germanen, Köln: Böhlau, 2008].

(https://www.hsozkult.de/review/id/reb-12254)

(https://edoc.hu-berlin.de/bitstream/handle/18452/18505/HistLit_2009-3.pdf)

5) H-Soz-Kult 22. März 2010 = Historische Literatur 8/1, 2010, S. 39-41 [zu Klaus Tausend, Im Inneren Germaniens. Beziehungen zwischen den germanischen Stämmen vom 1. Jh. v. Chr. bis zum 2. Jh. n. Chr., Stuttgart: Franz Steiner, 2009].

(https://www.hsozkult.de/review/id/reb-13782)

(https://edoc.hu-berlin.de/bitstream/handle/18452/18519/HistLit_2010-1.pdf)

6) H-Soz-Kult 14. November 2011 [zu Kai Ruffing (Hrsg.), Kontaktzone Lahn. Studien zum Kulturkontakt zwischen Römern und germanischen Stämmen, Wiesbaden: Harrassowitz, 2010].

(https://www.hsozkult.de/review/id/reb-16563)

7) Klio 93, 2011, S. 530-532 [zu Oliver Schipp, Der weströmische Kolonat von Konstantin bis zu den Karolingern, Hamburg: Dr. Kovač, 2009].

8) H-Soz-Kult 23. April 2012 [zu Histoire Auguste IV, 3. Vies des trente tyrans et de Claude. Texte établi, traduit et commenté par François Paschoud, Paris: Les Belles Lettres, 2011].

(https://www.hsozkult.de/review/id/reb-17108)

9) H-Soz-Kult 20. August 2012 [zu Gustav Adolf Lehmann, Imperium und Barbaricum, Wien: Verlag der Österreichischen Akademie der Wissenschaften, 2011].

(https://www.hsozkult.de/review/id/reb-17927)

10) H-Soz-Kult 15. Oktober 2012 [zu Gülden Cicekdagi, Publius Quinctilius Varus, Frankfurt a. M.: Peter Lang, 2012].

(https://www.hsozkult.de/review/id/reb-18223)

11) H-Soz-Kult 15. April 2013 [zu Thomas Fischer (Hrsg.), Die Krise des 3. Jahrhunderts n. Chr. und das Gallische Sonderreich, Wiesbaden: Reichert, 2012].

(https://www.hsozkult.de/review/id/reb-18895)

12) H-Soz-Kult 16. Dezember 2013 [zu Holger Sonnabend, August 14. Der Tod des Kaisers Augustus, Darmstadt: Wissenschaftliche Buchgesellschaft, 2013].

(https://www.hsozkult.de/review/id/reb-20124)

13) H-Soz-Kult 5. Mai 2014 [zu Michael Geiger, Gallienus, Frankfurt a. M.: Peter Lang, 2013].

(https://www.hsozkult.de/review/id/reb-20424)

14) Klio 96, 2014, S. 746-749 [zu Clifford Ando, Imperial Rome AD 193 to 284, Edinburgh: Edinburgh University Press, 2012]. 
15) H-Soz-Kult 7. April 2015 [zu Boris Dreyer, Orte der Varuskatastrophe und der römischen Okkupation in Germanien, Darmstadt: Konrad Theiss, 2014].

(https://www.hsozkult.de/review/id/reb-21964)

16) Historische Zeitschrift 302, 2016, S. 467-468 [zu Klaus Altmayer, Die Herrschaft des Carus, Carinus und Numerianus als Vorläufer der Tetrarchie, Stuttgart: Franz Steiner, 2014].

17) Sehepunkte 16/2, 2016 [zu Lorenzo Sguaitamatti, Der spätantike Konsulat, Fribourg: Academic Press Fribourg, 2012].

(http://sehepunkte.de/2016/02/21728.html)

18) H-Soz-Kult 18. September 2017 [zu Rudolf Aßkamp/Kai Jansen (Hrsg.), Triumph ohne Sieg. Roms Ende in Germanien, Darmstadt: Philipp von Zabern, 2017].

(https://www.hsozkult.de/review/id/reb-25929)

19) H-Soz-Kult 1. April 2019 [zu Günther Moosbauer, Die vergessene Römerschlacht. Der sensationelle Fund am Harzhorn, München: C. H. Beck, 2018].

(https://www.hsozkult.de/review/id/reb-27295)

20) H-Soz-Kult 20. Januar 2020 [zu Nikolas Hächler, Kontinuität und Wandel des Senatorenstandes im Zeitalter der Soldatenkaiser, Leiden: Brill, 2019].

(https://www.hsozkult.de/review/id/reb-28330)

In zweierlei Hinsicht ist das Schriftenverzeichnis zwar nicht lückenhaft, aber ausbaufähig. Das betrifft zum einen die Rezensionen zu den Werken Johnes, die vollkommen ausgeklammert wurden, aber gerade deswegen, da die einschlägigen bibliographischen Hilfsmittel in dieser Hinsicht (und insbesondere bei Forschungen aus der DDR) nicht immer Vollständigkeit aufweisen, von Interesse sind; aufgenommen wurden in den folgenden Listen nur die Rezensionen von Monographien und - aufgrund der großen Bedeutung des Werkes - dem Handbuch zur Soldatenkaiserzeit (die zu den Bänden, bei denen Johne Herausgeber und/oder Beiträger war, haben sich dagegen als weitgehend unergiebig erwiesen). Zum anderen handelt es sich um die Beiträge Johnes zu Lexika und Sammelwerken, die nur in sehr allgemeiner Form angegeben sind (S. 263-264).

Zunächst zu den Rezensionen. Hier sind neben dem Handbuch zur Soldatenkaiserzeit drei Monographien anzuführen (die S. 263 angeführte Druckfassung der Antrittsvorlesung Johnes scheint hingegen nicht rezensiert worden zu sein).

Kaiserbiographie und Senatsaristokratie (1976):

1) Jan Burian, Eirene 17, 1980, S. 145-146.

2) André Chastagnol, Revue historique 259, 1978, S. 77 mit Anm. 12.

3) André Chastagnol, Revue historique 269, 1983, S. 111-112 mit Anm. 10.

4) Alexander Demandt, Gnomon 50, 1978, S. 694-695.

5) Aristid I. Dovatur, Vestnik drevnej istorii 149, 1979, S. 196-202.

6) Roger P. H. Green, Journal of Roman Studies 69, 1979, S. 225-228 (hierzu S. 228).

7) Rigobert Günther, Zeitschrift für Geschichtswissenschaft 26, 1978, S. 653.

8) Rigobert Günther, Klio 61, 1979, S. 189-194 (hierzu S. 190-191).

9) Wieland Held, Deutsche Literaturzeitung 98, 1977, Sp. 730-733.

10) Leszek Mrozewicz, Eos 66, 1978, S. 179-181.

11) François Paschoud, Revue des études latines 55, 1977, S. 517-518.

12) Marie-Thérèse Raepsaet-Charlier, L’antiquité classique 47, 1978, S. 649-650.

13) Ronald Syme, Propaganda in the Historia Augusta, Latomus 37, 1978, S. 173-192 
= Ronald Syme, Historia Augusta papers, Oxford: Clarendon Press, 1983, S. 109-130.

Die Kolonen in Italien und den westlichen Provinzen (1983):

1) Jan Burian, Eirene 23, 1986, S. 132-134.

2) Luigi Capogrossi Colognesi, Labeo 33, 1987, S. 330-338.

3) André Chastagnol, Revue historique 280, 1988, S. 137-138 mit Anm. 192.

4) Alexander Demandt, Gnomon 57, 1985, S. 661-663.

5) Richard P. Duncan-Jones, Journal of Roman Studies 76, 1986, S. 295-297 (hierzu S. 297).

6) Hagen Fischer, Jahrbuch für Wirtschaftsgeschichte 1985/4, S. 167-175.

7) Bruce W. Frier, Zeitschrift der Savigny-Stiftung für Rechtsgeschichte 115, Romanistische Abteilung 102, 1985, S. 564-569.

8) Antonio Guarino, Labeo 30, 1984, S. 232 (Nr. 2).

9) Rigobert Günther, Deutsche Literaturzeitung 106, 1985, Sp. 174-176.

10) François Hinard, Revue historique 279, 1988, S. 131-132.

11) Liselot Huchthausen, Klio 67, 1985, S. 618-623.

12) Georgij S. Knabe, Vestnik drevnej istorii 183, 1987, S. 164-181 (Sammelrezension).

13) Marcel Morabito, Revue historique de droit français et étranger 4.S. 66, 1988, S. 118.

14) François Paschoud, Revue des études latines 62, 1984, S. 510-512.

15) Georges Raepsaet, Lantiquité classique 56, 1987, S. 468-469.

16) Bernhard Rink, Zeitschrift für Geschichtswissenschaft 32, 1984, S. 829.

17) Elena M. Štaerman, Listy filologické 109, 1986, S. 247-250.

18) Jean A. Straus, Latomus 46, 1987, S. 642-644.

19) Joachim Thiel, German Studies. Section 1. Philosophy and history 19, 1986, S. 59-61.

20) Ladislav Vidman, Listy filologické 108, 1985, S. 119-120.

21) Gerda von Bülow, Ethnographisch-archäologische Zeitschrift 28, 1987, S. 525-527.

Die Römer an der Elbe (2006):

1) Anthony R. Birley, Lantiquité classique 77, 2008, S. 736-737.

2) John F. Drinkwater, Latomus 68, 2009, S. 1090-1092.

3) Michael Erdrich, Germania 86, 2008, S. 791-795.

4) Hans-Werner Goetz, Anzeiger für die Altertumswissenschaft 62, 2009, Sp. 113-115.

5) Ulrich Lambrecht, Sehepunkte 7/5, 2007. (http://sehepunkte.de/2007/05/11539.html)

6) Yann Le Bohec, Revue des études latines 84, 2006, S. 420-421.

7) Rainer Wiegels, Bonner Jahrbücher 206, 2006, S. 371-373.

8) Christian Winkle, Gymnasium 114, 2007, S. 492-493.

9) Reinhard Wolters, Historische Zeitschrift 287, 2008, S. 157-158.

Die Zeit der Soldatenkaiser (2008):

1) Ferenc Barna, Acta classica universitatis scientiarum Debreceniensis 48, 2012, S. 191-205

2) Guido M. Berndt, Göttinger Forum für Altertumswissenschaft 14, 2011, S. 1087-1100. (http://gfa.gbv.de/z/2011/dr,gfa,014,2011,r,13)

3) Anthony R. Birley, Lantiquité classique 79, 2010, S. 673-675.

4) Jean-Marc Doyen, Bulletin du cercle détudes numismatiques 50, 2013, S. 172-177.

5) John F. Drinkwater, Journal of Roman Studies 100, 2010, S. 342-343.

6) Lily Grozdanova, Archaeologia bulgarica 13/3, 2009, S. 85-89. 
7) Matthias Haake, H-Soz-Kult 23. März 2009 = Historische Literatur 7/1, 2009, S. 19-21. (www.hsozkult.de/publicationreview/id/reb-12117)

(https://edoc.hu-berlin.de/bitstream/handle/18452/18503/HistLit_2009-1.pdf)

8) Katrin Herrmann, Gymnasium 117, 2010, S. 402-404.

9) Christian Körner, Sehepunkte 9/5, 2009.

(http://sehepunkte.de/2009/05/15201.html)

10) Ulrich Lambrecht, Das historisch-politische Buch 56, 2008, S. 599-600.

11) Laura Mecella, Mediterraneo antico 11, 2008, S. 657-671.

12) Karin Mosig-Walburg, Klio 92, 2010, S. 244-245.

13) François Paschoud, Antiquité Tardive 17, 2009, S. 403-405.

14) Marcus Reuter, Plekos 11, 2009, S. 109-112.

(http://www.plekos.uni-muenchen.de/2009/r-johne.pdf)

15) Michael Sommer, Zeitschrift für Geschichtswissenschaft 57, 2009, S. 641-643.

16) [S. n.], Helvetia archaeologica 40, 2009, S. 126.

An kleineren Beiträgen in Sammelwerken, die nicht einzeln im Schriftenverzeichnis erfasst sind, wären die Übersetzung und Kommentierung einiger Quellentexte in der Sammlung der Quellen zur Frühgeschichte Mitteleuropas (S. 263) sowie die Artikel in insgesamt vier Lexika zu nennen. Beim Lexikon der Antike sind angesichts der zahlreichen Änderungen sowohl die Artikel Johnes der ersten (1972) wie die der zehnten Auflage (1990) angeführt.

Für die Quellensammlung hat Johne insgesamt vier Autoren übersetzt und kommentiert.

1) Mit Hansulrich Labuske, „Caesar“, in: Joachim Herrmann (Hrsg.), Griechische und lateinische Quellen zur Frühgeschichte Mitteleuropas bis zur Mitte des 1. Jahrtausends u.Z. I, Berlin: Akademie-Verlag, 1988, S. 86-165 (Text und Übersetzung) und S. 450-474 (Kommentar).

2) „Tacitus, Annalen“, in: Joachim Herrmann (Hrsg.), Griechische und lateinische Quellen zur Frühgeschichte Mitteleuropas bis zur Mitte des 1. Jahrtausends u.Z. III, Berlin: Akademie-Verlag, 1991, S. 94-161 (Text und Übersetzung) und S. 506-532 (Kommentar).

3) Mit Renate Johne, „Cassius Dio“, in: Joachim Herrmann (Hrsg.), Griechische und lateinische Quellen zur Frühgeschichte Mitteleuropas bis zur Mitte des 1. Jahrtausends u.Z. III, Berlin: Akademie-Verlag, 1991, S. 266-335 (Text und Übersetzung) und S. 602-624 (Kommentar).

4) „Historia Augusta“, in: Joachim Herrmann (Hrsg.), Griechische und lateinische Quellen zur Frühgeschichte Mitteleuropas bis zur Mitte des 1. Jahrtausends u.Z. IV, Berlin: Akademie-Verlag, 1992, S. 188-221 (Text und Übersetzung) und S. 487-504 (Kommentar).

Mit Blick auf die Fachlexika ist von Interesse, dass die Schwerpunkte bei den einzelnen Artikeln Johnes variieren: In den späteren Auflagen des Lexikon der Antike - in den früheren stammen zu wenige Artikel von ihm, als dass eine klare Richtung erkennbar wäre - behandelt Johne die unterschiedlichsten Persönlichkeiten der Kaiserzeit vom ersten bis zum fünften Jahrhundert, im Lexikon früher Kulturen deuten die wenigen Artikel eher einen militärgeschichtlichen Schwerpunkt an, die Beiträge Johnes in der PIR sind vor allem für das dritte Jahrhundert von Bedeutung, während er als Beiträger für das Lexikon Der Neue Pauly vor allem zum fünften Jahrhundert gearbeitet hat. 
1a) Lexikon der Antike (1972, sieben Artikel): 1. Cäsarenwahnsinn; 2. Drakon; 3. Drusus; 4. Eugenius; 5. Hermunduren; 6. Scriptores Historiae Augustae; 7. Tacitus (2).

1b) Lexikon der Antike (1990, 145 Artikel und drei Listen): 1. Adoptivkaiser; 2. Agonistiker; 3. Agricola (1); 4. Agrippa; 5. Agrippina; 6. Aliso; 7. Antoninus Pius; 8. Arbogast; 9. Arcadius; 10. Augusta; 11. Aurelianus; 12. Avidius Cassius; 13. Bataver; 14. Belger; 15. Bonn; 16. Boudicca; 17. Brigetio; 18. Brukterer; 19. Caesarea; 20. Caligula; 21. Caracalla; 22. Carinus; 23. Carnuntum; 24. Carus; 25. Cäsarenwahnsinn; 26. Cassius Dio; 27. Chatten; 28. Chauken; 29. Cherusker; 30. Commodus; 31. Constans; 32. Constantinus; 33. Constantius; 34. Decuriones; 35. Didius Iulianus; 36. Diocletianus; 37. Diokletian; 38. Domitianus; 39. Drakon; 40. Drusus; 41. Eburacum; 42. Elagabalus; 43. Eugenius; 44. Eutropius; 45. Faustina; 46. Festus; 47. Flavius; 48. Florus; 49. Galba; 50. Galerius; 51. Gallienus; 52. Gallisches Sonderreich; 53. Germanen; 54. Germania; 55. Germanicus; 56. Geten; 57. Gordianus; 58. Gratianus; 59. Hadrianus; 60. Hadrumetum; 61. Hermunduren; 62. Heruler; 63. Honorius; 64. Iberer; 65. Illyrien; 66. Indiktion; 67. Ingenuus; 68. Ingwäonen; 69. Insubrer; 70. Iovianus; 71. Katalaunische Felder; 72. Konstantin; 73. Licinius; 74. Livia; 75. Londinium; 76. Macrinus; 77. Marbod; 78. Marcus Aurelius; 79. Mark Aurel; 80. Markomannen; 81. Maxentius; 82. Maximianus; 83. Maximinus Thrax; 84. Maximinus Daia; 85. Mediolanum; 86. Naissus; 87. Nero; 88. Nerva; 89. Nisibis; 90. Norden, Eduard; 91. Numerianus; 92. Obsequens; 93. Octavia; 94. Octavianus; 95. Odaenathus; 96. Odoaker; 97. Optimaten; 98. Otho; 99. Pächter; 100. Pertinax; 101. Pescennius Niger; 102. Philippus Arabs; 103. Postumus; 104. Quaden; 105. Ravenna; 106. Regensburg; 107. Ricimer; 108. Romulus Augustulus; 109. Scriptores Historiae Augustae; 110. Segestes; 111. Seianus; 112. Semnonen; 113. Septimius Severus; 114. Serdica; 115. Severus; 116. Severus Alexander; 117. Siegernamen; 118. Sirmium; 119. Soldatenkaiser; 120. Spätantike; 121. Stilicho; 122. Sueben; 123. Suetonius; 124. Sulpicius; 125. Syagrius; 126. Tetricus; 127. Themistios; 128. Theodosius; 129. Tiberius; 130. Titus; 131. Traianus; 132. Trebonianus Gallus; 133. Ulpius; 134. Valens; 135. Valentinianus; 136. Valerianus; 137. Vandalen; 138. Varus; 139. Verus; 140. Vespasianus; 141. Victor, Aurelius; 142. Vitellius; 143. Weströmisches Reich; 144. Xanten; 145. Zenobia; L1. Die wichtigsten hellenistischen Herrscherfamilien, S. 649; L2. Die römischen Kaiser, S. 650-651; L3. Stammtafeln der römischen Kaiserfamilien, S. 652-655.

2) Lexikon früher Kulturen (1984, fünf Artikel): 1. Heerwesen; 2. Kriegskunst; 3. Optimaten; 4. Suffeten; 5. Valentinianus.

3a) PIR V, 3 (1987, Buchstabe O, 88 Artikel): 1-5) Nr. 9-13 (S. 408-412); 6-7) Nr. 15-16 (S. 412-413); 8) Nr. 21 (S. 415); 9) Nr. 25 (S. 415-418); 10) Nr. 28 (S. 418-419); 11-14) Nr. 30-33 (S. 419); 15-19) Nr. 36-40 (S. 421-422); 20) Nr. 48 (S. 424); 21-23) Nr. 50-52 (S. 425); 24) Nr. 54 (S. 426); 25) Nr. 56 (S. 426); 26-28) Nr. 59-61 (S. 428); 29-30) Nr. 63-64 (S. 429); 31) Nr. 67 (S. 433); 32-36) Nr. 69-73 (S. 433-434); 37-38) Nr. 76-77 (S. 434-435); 39) Nr. 79 (S. 435); 40) Nr. 83 (S. 436); 41-44) Nr. 85-88 (S. 436-437); 45) Nr. 93 (S. 440); 46) Nr. 98 (S. 440-441); 47-48) Nr. 100-101 (S. 441); 49) Nr. 103 (S. 441-442); 50-53) Nr. 106-109 (S. 442-450); 54) Nr. 112 (S. 450-451); 55) Nr. 115 (S. 452); 56-57) Nr. 119-120 (S. 453); 58) Nr. 129 (S. 457-458); 59) Nr. 133 (S. 459); 60-65) Nr. 140a-145 (S. 461-463); 66) Nr. 147 (S. 463); 67) Nr. 149 (S. 463); 68-69) Nr. 154-155 (S. 464); 70-73) Nr. 157-160 (S. 465-466); 74-75) 
Nr. 168-169 (S. 470-471); 76-77) Nr. 176-177 (S. 472-473); 78) Nr. 181 (S. 478); 79-88) Nr. 183-192 (S. 479-484).

3b) PIR VI (1998, Buchstabe P, 86 Artikel): 1) Nr. 1 (S. 1); 2) Nr. 842 (S. 357-358); 3) Nr. 851 (S. 365); 4) Nr. 854 (S. 366); 5) Nr. 857 (S. 367); 6-7) Nr. 861-862 (S. 368); 8) Nr. 864 (S. 369); 9) Nr. 868 (S. 369); 10) Nr. 872 (S. 370); 11) Nr. 877 (S. 371-372); 12) Nr. 882 (S. 373); 13-14) Nr. 887-888 (S. 376); 15-21) Nr. 890-896 (S. 376-378); 22-24) Nr. 898-900 (S. 378-380); 25-27) Nr. 902-904 (S. 381); 28-29) Nr. 906-907 (S. 381-382); 30) Nr. 911 (S. 382); 31) Nr. 913 (S. 382-383); 32) Nr. 919 (S. 386); 33-34) Nr. 921-922 (S. 387); 35) Nr. 925 (S. 388); 36) Nr. 928 (S. 390-391); 37-39) Nr. 941-943 (S. 398-399); 40) Nr. 950 (S. 401); 41) Nr. 952 (S. 402); 42-43) Nr. 959-960 (S. 404); 44) Nr. 965 (S. 404-405); 45-46) Nr. 970-971 (S. 405); 47-51) Nr. 973-977 (S. 406-411); 52) Nr. 979 (S. 411); 53-54) Nr. 995-996 (S. 416); 55) Nr. 1001 (S. 418); 56) Nr. 1004 (S. 418); 57-58) Nr. 1008-1009 (S. 425-426); 59-60) Nr. 1014-1015 (S. 427-428); 61) Nr. 1019 (S. 428-429); 62) Nr. 1034 (S. 432); 63) Nr. 1039 (S. 433); 64) Nr. 1041 (S. 433); 65-67) Nr. 1043-1045 (S. 435); 68-70) Nr. 1050-1052 (S. 437); 71) Nr. 1055 (S. 438); 72-73) Nr. 1057-1058 (S. 439); 74-76) Nr. 1064-1066 (S. 440); 77) Nr. 1070 (S. 441); 78) Nr. 1074 (S. 443); 79-81) Nr. 1077-1079 (S. 444); 82-84) Nr. 1084-1086 (S. 445-446); 85) Nr. 1092 (S. 449); 86) Nr. 1096 (S. 449-450).

4) Der Neue Pauly (1996-2002, 54 Artikel): 1. Aegidius; 2. Agrippinus; 3. Anthemios/ -us (2); 4. Arbogastes; 5. Avitus (1); 6. Faustus (1); 7. Faustus (2); 8. Felix (6); 9. Ferreolus; 10. Festus (5); 11. Fidelis; 12. Flavianus (3); 13. Florentius (3); 14. Florentius (4); 15. Gabrielus; 16. Gaiso (2); 17. Gaudentios/-us (4); 18. Gerontius (3); 19. Gildas; 20. Glycerius; 21. Heraclianus; 22. Herakleios (5); 23. Herculius (1); 24. Historia Augusta; 25. Honoria; 26. Honorius (3); 27. Iovinus (2); 28. Iovius (2); 29. Isidoros/us (5); 30. Iustus/-os (3); 31. Lagodius; 32. Laterculum; 33. Litorius; 34. Longinianus; 35. Macedonius (2); 36. Magnus (6); 37. Magnus (7); 38. Nomus; 39. Notitia Africae; 40. Notitia Dignitatum; 41. Notitia Galliarum; 42. Notitia Romae; 43. Notitia urbis Constantinopolitanae; 44. Parthenius (2); 45. Parthenius (3); 46. Priscus (3); 47. Regendarius; 48. Reparatus (1); 49. Rufinus (1); 50. Ulpius (3); 51. Ulpius (4); 52. Vigilantia; 53. Zemarchos; 54. Zenonis.

Zum Schluss noch einige Anregungen im Bereich der Wissenschaftsgeschichte. Die Frage, die sich dabei am stärksten aufdrängt, ist die nach der Verbindung der Person und der Forschungen Johnes (zumal bei einem Thema wie dem Kolonat) mit dem DDRRegime. Eine zuverlässige Untersuchung zum erstgenannten Aspekt erfordert eine eingehende Prüfung des Archiv- und Aktenmaterials, ${ }^{11}$ doch zum zweiten lassen sich auf

11 Eine noch immer wichtige allgemeine Vorarbeit ist die Dissertation von Matthias Willing, Althistorische Forschung in der DDR, Berlin: Duncker \& Humblot, 1991. Johne wird dort (im Gegensatz etwa zu seinem akademischen Lehrer Seyfarth: S. 142-144) zwar nicht systematisch behandelt, jedoch immer wieder in verschiedenen Zusammenhängen erwähnt (siehe den Eintrag im Registerteil S. 309). Speziell zur Geschichte der PIR (und somit auch zur Tätigkeit Johnes) handelt jetzt auch Werner Eck, „Die PIR im Spiegel der beteiligten Personen“, in: Werner Eck, Matthäus Heil (Hrsg.), Prosopographie des Römischen Kaiserreichs, Berlin: Walter De Gruyter, 2017, S. 1-94 (zu Johne: S. 1, Anm. 1; S. 15, Anm. 46; S. 44 mit Anm. 161; S. 52 mit Anm. 195; S. 57 mit Anm. 217; S. 58, Anm. 221; S. 59; S. 65, 
Basis des in dem Band und diesem Beitrag gebotenen Materials einige erste Grundlagen erarbeiten.

Eine Durchsicht der oben angeführten Rezensionen zu seinen beiden Monographien (außer den mir sprachlich unzugänglichen) ${ }^{12}$ ergab ein fast durchgehend positives Urteil. Die einzige stark negative gehaltene Kritik an seinem Buch zur Historia Augusta stammt von Ralph Green, der es als „rather pedestrian“ und „only a minimal contribution to the subject“ bezeichnet und über Johne meint, seine „conclusions are unoriginal, his methods unrefined, and his capacity for doxography apparently unlimited“ (alle Zitate Journal of Roman Studies 69, 1979, S. 228). Der Kritik, die das Urteil zudem nicht anhand von Einzelfragen näher begründet, kann mit Blick auf das deutlich positivere Urteil der zahlreichen Rezensenten, die sich in größerem Ausmaß mit der Historia Augusta befasst haben (darunter immerhin André Chastagnol, Alexander Demandt und François Paschoud), keine große Bedeutung beigemessen werden - hier ist die Diskussion einer Reihe von Einzelfragen durch Ronald Syme (Latomus 37, 1978, S. 173-192 = Historia Augusta papers, Oxford: Clarendon Press, 1983, S. 109-130) deutlich relevanter -, aber wichtig für die hier diskutierte Frage ist die Tatsache, dass auch ein so harscher Kritiker offensichtlich keine ideologisch begründete Problematik des Buches ermitteln konnte. Aus der anderen Richtung ist festzustellen, dass die DDR-Forschung (namentlich Rigobert Günther und Wieland Held) das Buch ebenfalls wohlwollend aufnahm und sein fachlicher Wert offensichtlich die mangelnde ideologische Ausrichtung ausgleichen konnte, so dass entsprechende Kritik durch Wieland Held nur sehr angedeutet und vorsichtig geäußert wurde. ${ }^{13}$

Nun könnte man das weitgehend positive Urteil über die Dissertation Johnes damit erklären, dass die Altertumswissenschaft in der DDR zwar ein großes Interesse an den in der Historia Augusta geschilderten Ereignissen, dafür aber nur ein vergleichsweise geringes an dem Werk selbst und den damit verbundenen großen Fragen (Datierung, Autorschaft, Tendenz) aufweist ${ }^{14}$ und Johnes Erstlingswerk sich somit auf insgesamt unproblematischem Terrain bewegte. Allerdings fällt auf, dass sich auch für die Publi-

Anm. 254; S. 67 mit Anm. 264-265; S. 68, Anm. 268-269; S. 69 mit Anm. 271; S. 70, Anm. 275; S. 73, Anm. 286).

12 Konkret sind das zur Dissertation Johnes die von Dovatur und Mrozewicz und zu seiner Habilitationsschrift die von Knabe, Štaerman und Vidman. Es wäre ohne Zweifel ein lohnenswertes Unterfangen, wenn Forscher mit entsprechenden Sprachkenntnissen diese und vergleichbare Beiträge systematisch durchgehen und die daraus resultierenden Ergebnisse für die Altertumswissenschaft wie für die Wissenschaftsgeschichte einem größeren Fachpublikum zugänglich machen würden. Bislang werden, wie es scheint, Rezensionen als Quelle für wissenschaftsgeschichtliche Fragen auch allgemein noch immer nur unzureichend ausgewertet.

13 Deutsche Literaturzeitung 98, 1977, Sp. 732: „Sicher wäre es wünschenswert gewesen, wenn J. es unternommen hätte, im Laufe seiner Untersuchungen bzw. zumindest am Schluß seiner Studie auf die Rolle der stadtrömischen Senataristokratie zu verweisen, die diese in der Übergangszeit von der auf Sklaverei beruhenden Gesellschaftsformation zum Feudalismus spielte“.

14 Als Beispiel für die Vorgehensweise und die Priotitäten stärker ideologisch beeinflusster Forscher sei auf die Dissertation von Hans-Joachim Herrmann verwiesen: Studien über Usurpationen und Krise im 3. Jahrhundert u. Z. unter besonderer Berücksichtigung ihrer Darstellung in der „Historia Augusta“, Diss., Greifswald, 1964. Herrmann diskutiert zwar das Problem der Historia Augusta etwas ausführlicher, lehnt aber sämtliche Überlegungen seit Dessau ohne eingehendere Auseinandersetzung mit selbigen als unbewiesene Hypothesen ab (lediglich einige spätere Eingriffe eines späteren Redaktors lässt er gelten) und stellt S. 64 fest: „Das Problem der Tendenz kann nicht lediglich im Sinne einer konkreten politischen Propaganda, die nur aus einer zeitlich exakt festzulegenden nachkonstantinischen Situation erklärbar ist, erörtert werden. Es muß als Widerspiegelung bestimmter Klasseninteressen - auch des 3. Jahrhunderts - umfassender analysiert werden. "Entgegen dem, was der Titel der 
kationsfassung der Habilitationsschrift, die mit dem Kolonat ein für die DDR-Ideologie deutlich interessanteres Thema behandelte, der Befund nicht ändert. Die westlichen Wissenschaftler, die sich zu dem Buch äußerten, kamen zu einem klar positiven Urteil, ${ }^{15}$ aber auch die Kritiker aus Ländern des Ostblocks nahmen das Buch wohlwollend an. Der Schlüssel zum Verständnis dessen liegt weniger in dem Thema selbst, sondern vielmehr in der Art der Aufbereitung (nicht nur) durch Johne: Im Wesentlichen handelt es sich bei dem 1983 publizierten Buch um eine Sammlung der relevanten Passagen aus den antiken Quellenwerken, die in Text, Übersetzung und einem Sachkommentar geboten werden, nicht so sehr hingegen um eine systematische Erfassung des Kolonats als Phänomen im Kontext der römischen Sozial- und Wirtschaftsgeschichte. ${ }^{16}$ Bei den weiteren Studien Johnes aus DDR-Zeiten zu dem Thema Kolonat handelt es sich entweder um Kurzfassungen der Inhalte des Buches, um extrem speziell gehaltene Untersuchungen einzelner Passagen der Historia Augusta (S. 187-196) oder um terminologische Untersuchungen, in denen der Bedeutung des Begriffs in der Antike nachgegangen wird und somit eher um philologische als um sozial- und wirtschaftsgeschichtliche Beiträge im engeren Sinne (S. 149-164 und S. 181-185).

Der Gesamteindruck, wie er sich aus dem publizierten Schrifttum Johnes und den Schilderungen der Kurzbiographie Hartmanns zu Beginn des Bandes (insbesondere S. 11-12) ergibt, ist also der folgende: Johne gelang es, mit seinen Forschungsthemen bzw. deren praktischer Umsetzung eine Nische zu finden, die ihn einerseits mangels Interesse des Regimes daran nicht dazu zwang, sich in seinen Publikationen in einer der wissenschaftlichen Integrität entgegenstehenden Weise den ideologischen Vorgaben der SED zu unterwerfen, ihm andererseits aber im In- und Ausland ausreichend fachliches Ansehen einbrachte, um seine Forschungen nicht nur ungehindert, sondern auch mit ausreichender Förderung fortsetzen zu können. Dabei mag gewiss auch der finanzielle Aspekt eine Rolle gespielt haben, denn die Fortführung und der Verkauf unverzichtbarer Standardwerke wie der PIR brachte der DDR neben wissenschaftlichem Ansehen auch zusätzliche Geldmittel. Bis eingehende Archiv- und Aktenstudien vorliegen, lässt das erfasste und publizierte Material die folgende Einschätzung am plausibelsten erscheinen: Johne war weder ein begeisterter Anhänger des DDR-Systems noch ein offener Gegner desselben, sondern gehörte zu denjenigen Forschern, denen es gelang, sich mit den Gegebenheiten zu arrangieren, ohne dabei bewusst oder auch nur unbewusst ihre Prinzipien wissenschaftlichen Arbeitens aufzugeben.

Die auf den vorhergehenden Seiten gebotenen umfangreichen Ausführungen haben nun hoffentlich nicht den Eindruck erweckt, der Band sei insgesamt ein nur mangelhaftes Werk. Gewiss, er ist in mancherlei Hinsicht noch ausbaufähig und hat an einigen weni-

Dissertation und das angeführte Zitat versprechen, ergeben sich für die Historia Augusta als Werk aus den weiteren Ausführungen Herrmanns keine weiteren Erkenntnisse irgendwelcher Art.

15 Paschouds letzter Satz in seiner Rezension lautet sogar (Revue des études latines 62, 1984, S. 512): "Ce livre ne devrait donc manquer dans aucune bonne bibliothèque du domaine classique." Die jüngste Gesamtbewertung bietet Oliver Schipp, Der weströmische Kolonat von Konstantin bis zu den Karolingern (332 bis 861), Hamburg: Dr. Kovač, 2009, S. 1-2 mit Anm. 3, der zwar die marxistische Grundtendenz des Buches hervorhebt, diese jedoch vor allem im (nicht von Johne stammenden) juristischen Teil verortet.

16 Darauf wies bereits Antonio Guarino in seiner kurzen Anzeige hin (Labeo 30, 1984, S. 232): „Lopera non difende nuove e originali teorie sulla genesi e sugli sviluppi di uno dei piú oscuri istituti dellesperienza romana, ma appresta una ricca dotazione di dati e di dottrine agli studiosi futuri“. 
gen Stellen sogar starken Verbesserungsbedarf (vor allem mit Blick auf die Querverweise und das fehlende Register). Doch die wesentliche Leistung Johnes in seinem Gesamtwerk, die erneute und stellenweise erheblich verbesserte Zugänglichmachung desselben durch den Neuabdruck der Aufsätze und das Schriftenverzeichnis haben gegenüber den problematischen Elementen ein deutliches Übergewicht. Die Aufsätze Johnes waren zum Zeitpunkt ihrer Publikation von großem Wert und sind das auch jetzt noch - außer in den Fällen, wo sie durch seine eigenen Forschungen zum selben Thema durch bessere Darstellungen ersetzt sind. Jeder Forscher, der sich mit den in dem Band behandelten Themen, insbesondere der Historia Augusta und/oder der Zeit der Soldatenkaiser, befasst, wird den Band als nützliches Arbeitsinstrument zu schätzen wissen.

\title{
VOJENŠTÍ CÍSAŘI, SCRIPTORES HISTORIAE AUGUSTAE \\ A PŘIPOUTÁNÍ K PŮDĚ. \\ POZNÁMKY KE KNIZE KAISER, KONSULN UND KOLONEN \\ KLAUSE-PETRA JOHNEHO (2007) \\ A K JEHO HISTORICKÉMU DÍLU JAKO CELKU
}

Př́íspěvek podrobně rozebírá soubor drobných spisů známého historika starověkých dějin Klause-Petra Johneho publikovaný v roce 2007. Kromě shrnutí obsahu jednotlivých článků a rozboru tezí, které jsou v nich podány, je předložena zejména řada doplňků k úplnému soupisu Johneho díla, který je cenný coby bibliografický zdroj. S tím se pojí některé obecné metodické úvahy o konceptu souboru článků a stručné zhodnocení Johneho bádání v rámci pojetí věd o starověku v Německé demokratické republice.

\author{
Raphael Brendel \\ Ludwig-Maximilians-Universität München \\ raphaelbrendel@arcor.de
}

